\title{
Modeling Failure of 3D Fiber Reinforced Foam Core Sandwich Structures with Defects
}

\author{
Zachary T. Kier ${ }^{1}$ and Anthony M. Waas ${ }^{2}$ \\ University of Michigan, Ann Arbor, MI 48109 \\ and \\ Jacob I. Rome, Vinay K. Goyal, Patrick Schubel, Gary Steckel, Dhruv Patel, and Yong Kim \\ The Aerospace Corporation, El Segundo, CA 90245
}

\begin{abstract}
Foam core sandwich composites are widely used in primary structural components of launch vehicles and spacecraft. These structures exhibit complex failure modes that are sensitive to butt-joints, core mismatches, impact damage, voids and facesheet delaminations. 3D Fiber Reinforced Foam Core (3DFRFC) represents a new class of core material designed to replace standard foam core in future aerospace structures. An analysis, test, and nondestructive evaluation program was developed to estimate the strength reduction for debonds between the facesheet and the 3DFRFC. A compression test method was selected to evaluate fully bonded samples and samples with debonds of varying diameters: 0.5 inch, 1.0 inch, 1.5 inch, and 2.0 inch. The manufactured debonds were verified using throughtransmission ultrasonic inspection. Nonlinear finite element analysis with a progressive failure methodology was used to understand the failure processes and predict strength reduction. The typical failure mode for $0.5 \mathrm{inch}, 1.0 \mathrm{inch}$, and $1.5 \mathrm{inch}$ debonds was facesheet compression failure, while analysis showed that for larger debonds failure consisted of a rapid sequence of buckling, delamination, and fiber failure. Testing and analysis demonstrated significant strength reductions for the 2.0 inch debond.
\end{abstract}

\section{Introduction}

A current engineering challenge for composite sandwich structures is to quantify their ability to tolerate damage, particularly in launch vehicles and spacecraft where mission assurance is critical to mitigating cost from loss or failure. The strength of sandwich composites can be reduced through many mechanisms, including debonds between a facesheet and the core. Debonds of sufficient size, which are typically introduced during manufacturing of foam core composite sandwich structures, could become critical and lead to catastrophic failure. These debonds can result from impact damage, embedded foreign objects, use of poor bonding agents, or surface preparation issues.

Recently, new core materials have been developed that could affect the damage tolerance of sandwich composites. One class of core material being considered uses a three-dimensional, truss-like network of reinforcing fibers inside a lightweight foam core. Examples of this emerging class of core materials include NidaFusion ${ }^{1}$ and TYCOR $\AA^{2}$. The truss structure of the 3D fiber network provides added paths for load transfer and acts to impede crack propagation within the foam. As a result of the added load paths, the failure and strength of these threedimensionally reinforced sandwich composites become exceedingly difficult to predict. It is necessary to quantify this damage tolerance and develop methods that allow for the practical application of these materials.

This research aims to experimentally and analytically quantify the reduction in compression strength of 3D Fiber Reinforced Foam Core (3DFRFC) sandwich composites with debonds between the core and the facesheet. Due to the complexity of the 3DFRFC, the manufacturing, non-destructive evaluation (NDE), testing, and analysis program was first developed with a monolithic foam core.

\footnotetext{
${ }^{1}$ NASA Space Technology Research Fellow, Department of Aerospace Engineering, 1320 Beal Avenue, Ann Arbor, MI, 48109, AIAA Student Member, ztkier@umich.edu.

${ }^{2}$ Felix Pawlowski Collegiate Professor of Aerospace Engineering, Department of Aerospace Engineering, 1320 Beal Avenue, Ann Arbor, MI, 48109, AIAA Fellow.
}

American Institute of Aeronautics and Astronautics 


\section{Experimental and Analytical Investigation of Monolithic Foam Core}

The material system chosen for this investigation was IM7/8552 carbon epoxy for the facesheets and 0.75 inch 200WF Rohacell for the foam core. AF191 film adhesive was used to bond the facesheets to the core. Testing methods were selected based on an investigation of typical loads on a launch vehicle. This study found that in-plane compression was the dominant stress, and as a result the edgewise compression test was selected, ASTM C 364-99 ${ }^{3}$. This load case is of particular interest for the study of facesheet-core debonds because the compressive stress in the facesheet is capable of causing localized buckling of the unbonded region of the facesheet. The ASTM guidelines for specimen sizing were utilized to ensure that failure would not result from a global buckling mode. The specimens were designed to contain circular debonds of 0.5 inch, 1.0 inch, 1.5 inch, or 2.0 inch diameter, Figure 1. Debond sizes were selected based on typical criteria used in the launch industry. The desired debonds were manufactured by removing a circular region of adhesive and replacing it with a PTFE insert. The panels were inspected via NDE to ensure panel quality and to verify debond placement in the cured sandwich panel prior to removing the desired samples from the fabricated panels, Figure 2.

The composite sandwich coupons with varying sized defects were tested according to ASTM C 364-99 using an Instron Universal Testing Machine, Figure 3. The tests were conducted at a displacement rate of $0.2 \mathrm{in} / \mathrm{min}$ until catastrophic specimen failure. Strain gages were placed in the center of each facesheet. The load and axial displacement were recorded from the Instron while the transverse displacement was recorded near the center of the debond using an extensometer. Two or three samples were tested for each debond size.

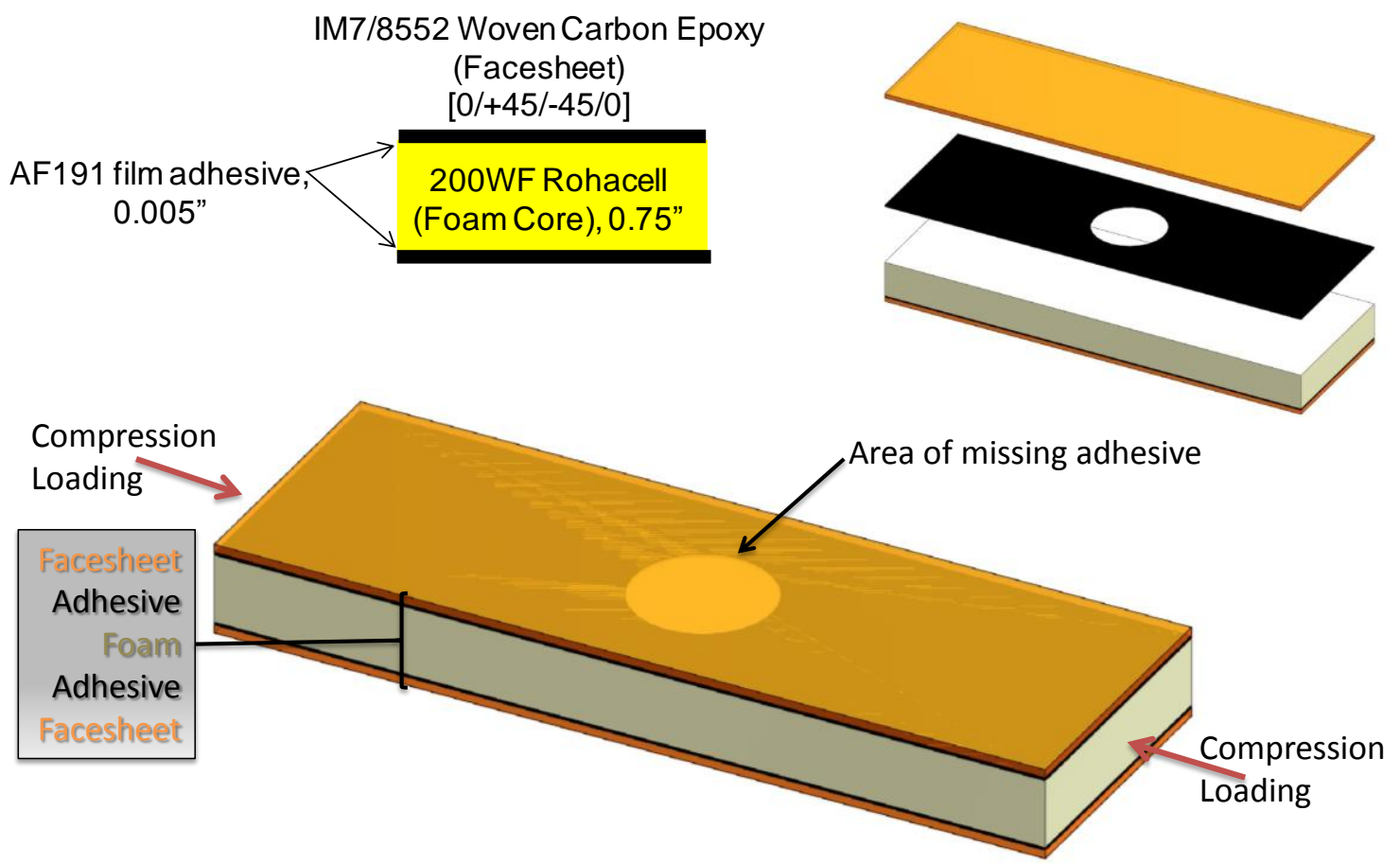

Figure 1. Schematic of core-facesheet debond specimen (not to scale). 

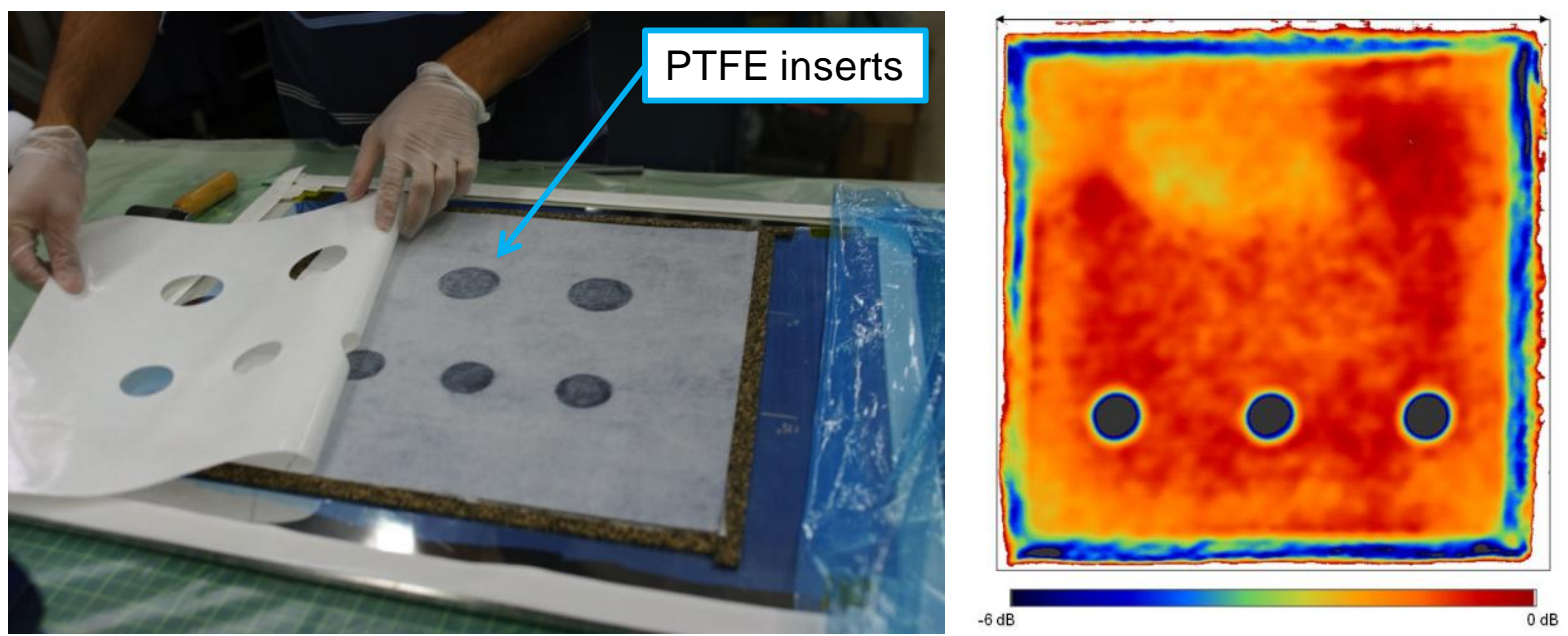

Figure 2. Film adhesive with 1.5 inch and 2.0 inch PTFE inserts (left) and ultrasonic transmission with 1.0 inch inserts (right).

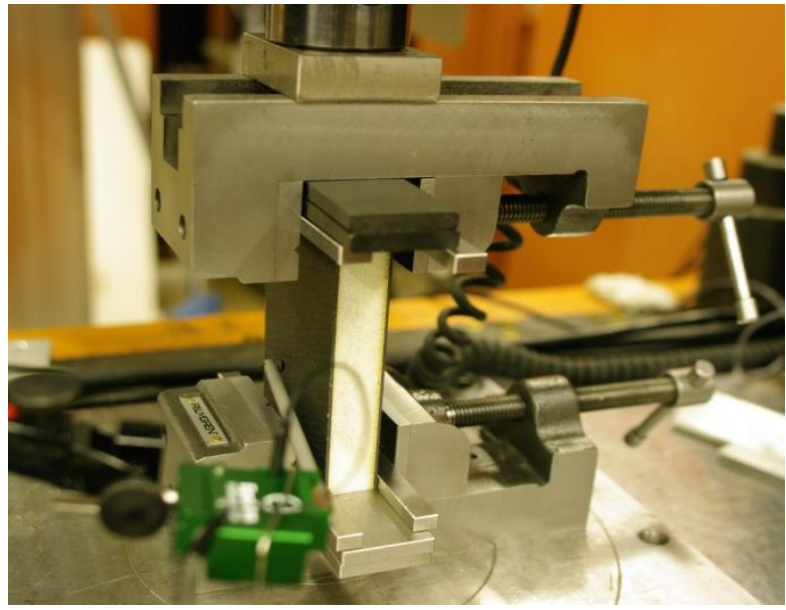

Figure 3. Edgewise compression test fixture.

The analytical evaluation was performed using finite element analysis. A quarter symmetry finite element model of the experimental setup was developed and solved using the finite element software Abaqus to evaluate debonds between the facesheet and the core. On the side of the sample with the debond, the facesheet plies were individually modeled with brick elements and the adhesive layer between the facesheet and the foam was modeled with Abaqus decohesion elements, Figure 4. The strength and fracture properties for the interface were based on measured foam properties, as the foam is the weaker of the constituents at the interface. The properties of the facesheet lamina, foam core and film adhesive are provided, Table I. The value for the lamina compression strength was determined from prior edgewise compression tests. The value used for the Mode I critical strain energy release rate of the Rohacell $200 \mathrm{WF}$ is an assumed lower bound based on preliminary fracture testing and is supported as a lower bound by published results ${ }^{4}$. Due to the lack of testing, the Mode II and Mode III fracture values for the foam were taken as equal to the mode I values for the analysis in the interest of maintaining a lower bound on the fracture properties. The remainder of the facesheet and Rohacell 200WF properties were measured through in-house testing ${ }^{5}$, while the mechanical properties of the film adhesive were obtained from vendor data ${ }^{6}$. 
Table I. Properties of sandwich constituent materials.

\begin{tabular}{|c|c|}
\hline \multicolumn{2}{|c|}{ Facesheet Lamina Properties, SGP370-8H/8552 } \\
\hline $\mathrm{E}_{1}(\mathrm{Msi})$ & 11.1 \\
\hline $\mathrm{E}_{2}(\mathrm{Msi})$ & 11.6 \\
\hline$v_{12}$ & 0.05 \\
\hline Tensile Strength $\mathrm{F}_{1 \mathrm{t}}(\mathrm{ksi})$ & 154 \\
\hline $\mathrm{F}_{2 \mathrm{t}}(\mathrm{ksi})$ & 150 \\
\hline Strain to Failure $\varepsilon_{1 \mathrm{t}}(\%)$ & 1.35 \\
\hline Compressive Strength $\mathrm{F}_{1 \mathrm{c}}(\mathrm{ksi})$ & 76.1 \\
\hline \multicolumn{2}{|c|}{ Foam Core Properties, Rohacell 200WF } \\
\hline Tensile Modulus, $\mathrm{E}_{\mathrm{t}}(\mathrm{ksi})$ & 56.0 \\
\hline Poisson's Ratio, $v$ & 0.35 \\
\hline Tensile Strength, $\mathrm{F}_{\mathrm{t}}(\mathrm{ksi})$ & 1.12 \\
\hline Shear Strength, $\mathrm{F}_{\mathrm{s}}(\mathrm{ksi})$ & 0.966 \\
\hline $\begin{array}{l}\text { Mode I Critical Strain Energy } \\
\text { Release Rate, } \mathrm{G}_{1 \mathrm{c}} \text { (lbf/inch) }\end{array}$ & 1.0 \\
\hline \multicolumn{2}{|c|}{ Film Adhesive Properties, AF191 } \\
\hline Elastic Modulus, E (ksi) & 320.0 \\
\hline Poisson's Ratio, $v$ & 0.40 \\
\hline
\end{tabular}

The failure load was predicted using a progressive failure methodology including nonlinear geometry. Displacement control was used to simulate test boundary conditions and enable the numerical simulation of failure progression. Progressive failure analysis (PFA) did not account for matrix-cracking and fiber failure, because it was observed in the experiment that the structural response was linear and failure of the samples occurred suddenly in the form of catastrophic facesheet compression failure. There was no indication that matrix-cracking preceded the ultimate failure, but most likely it occurred concurrently with the onset of fiber failure. While PFA was used to simulate delamination propagation, the facesheet compression failure was predicted by identifying the load at which the facesheet stress exceeded its compression strength.

Linear buckling analysis was performed for each defect size to determine critical buckling modes. Linear buckling analysis for a 0.5 inch circular debond showed that the critical buckling mode is global bending of the panel instead of local buckling of the unsupported debond, Figure 5. For defects larger than 1 inch, a localized buckle of the facesheet within the unbonded region was found to be critical. Subsequently, the modes from the linear buckling analysis were scaled to introduce a geometric imperfection into the nonlinear model. Analysis was also conducted to evaluate a 3 inch debond, although no test samples containing defects larger than 2 inches were fabricated due to the availability of material and limitations on the required specimen geometry. The analysis showed that for debonds larger than 1.5 inch, failure evolves into a multi-step failure occurs with facesheet buckling driving delamination propagation, eventually leading to facesheet compression failure, Figure 6. 

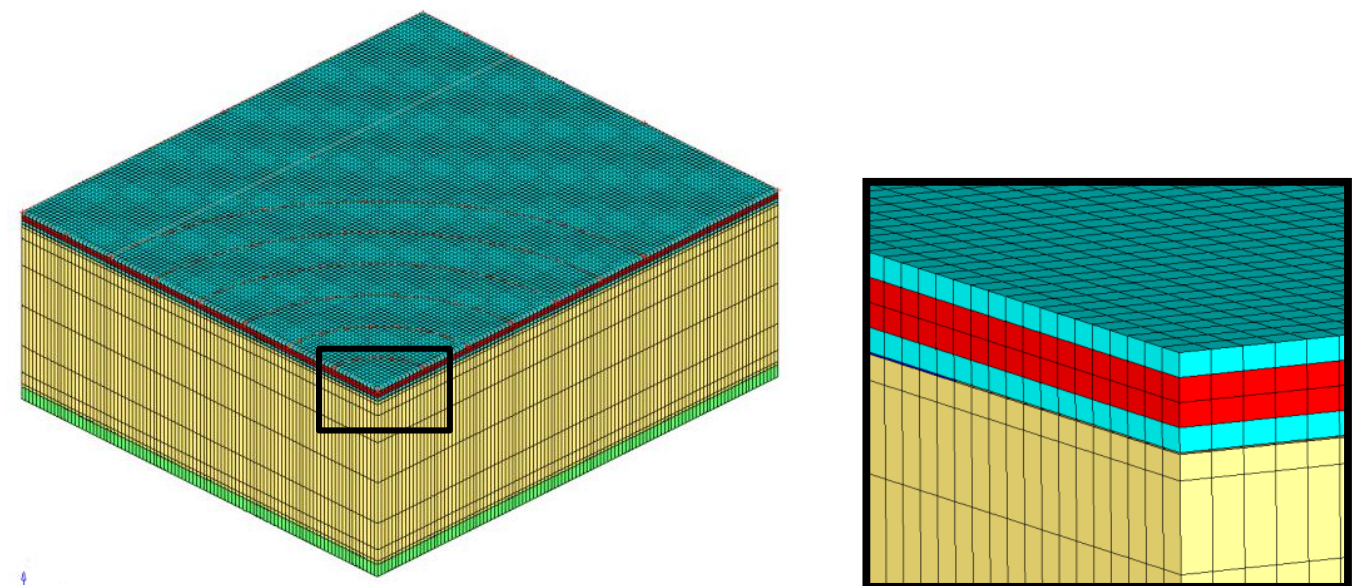

Figure 4. Quarter symmetry edgewise compression finite element model.

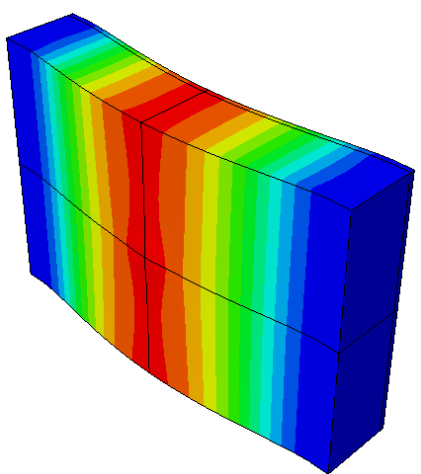

$1 / 2 "$ Debond

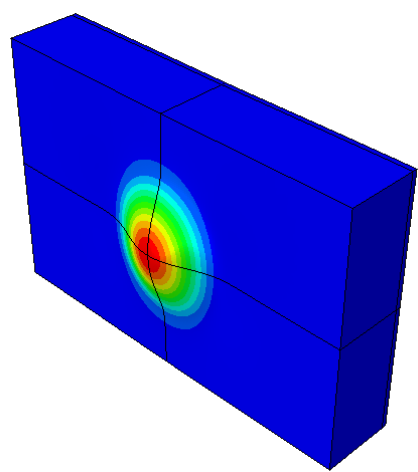

$\geq 1$ " Debond

Figure 5. Linear buckling modes for increasing debond size.

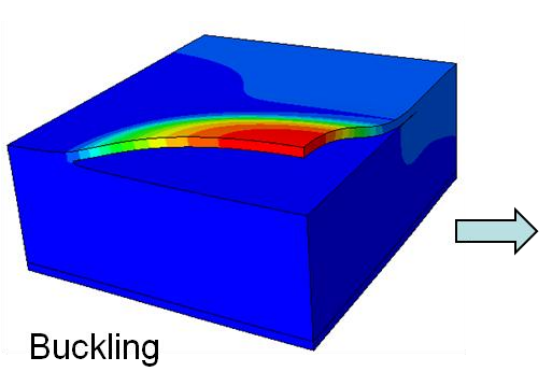

Figure 6. Failure progression for larger debonds.

The predicted strength of the edgewise compression samples was unaffected by the presence of 0.5 inch and 1.0 inch debonds. In these samples fiber failure occurs before the onset of buckling. The predicted strength of the remaining samples with debonds was reduced when compared to the sample without defect, especially for the 3.0 inch sample. Fiber failure was predicted to initiate with the onset of buckling for samples with $1.5 \mathrm{inch}$ and 2.0 inch debonds. Predicted failure for the 3.0 inch debond sample was driven by unstable growth of the debond. The analysis was within $10 \%$ of test data, Table II. It should be noted that the foam core utilized for the panel containing the no-debond, $0.5 \mathrm{inch}$, and $1.0 \mathrm{inch}$ debond samples experienced surface oxidation during the fabrication process. This oxidation resulted in a change in failure mode in these samples from facesheet compression to facesheet delamination, Figure 7. 
Table II. Observed and predicted strength reduction with facesheet-core debonds.

\begin{tabular}{|c|c|c|c|c|}
\hline $\begin{array}{c}\text { Debond } \\
\text { Diameter (inch) }\end{array}$ & $\begin{array}{c}\text { Test Facesheet } \\
\text { Stress (ksi) }\end{array}$ & $\begin{array}{c}\text { Nonlinear Failure } \\
\text { Prediction Facesheet } \\
\text { Stress (ksi) }\end{array}$ & $\begin{array}{c}\text { Strength } \\
\text { Reduction based } \\
\text { on Test (\%) }\end{array}$ & $\begin{array}{c}\text { Strength } \\
\text { Reduction based } \\
\text { on Analysis (\%) }\end{array}$ \\
\hline$-^{*}$ & $56.2^{*}$ & 57.5 & $2.4^{*}$ & -- \\
\hline $0.5^{*}$ & $56.8^{*}$ & 57.5 & $1.3^{*}$ & 0 \\
\hline $1.0^{*}$ & $54.9^{*}$ & 57.5 & $4.6^{*}$ & 0 \\
\hline 1.5 & 53.6 & 53.3 & 6.8 & 8.4 \\
\hline 2.0 & 51.6 & 45.7 & 10.3 & 21.5 \\
\hline 3.0 & N/A & 30.0 & N/A & 48.4 \\
\hline
\end{tabular}

*Foam core for these samples experienced surface oxidation during heat-treatment process, an undesirable outcome. Steps need to be taken to avoid foam oxidation.

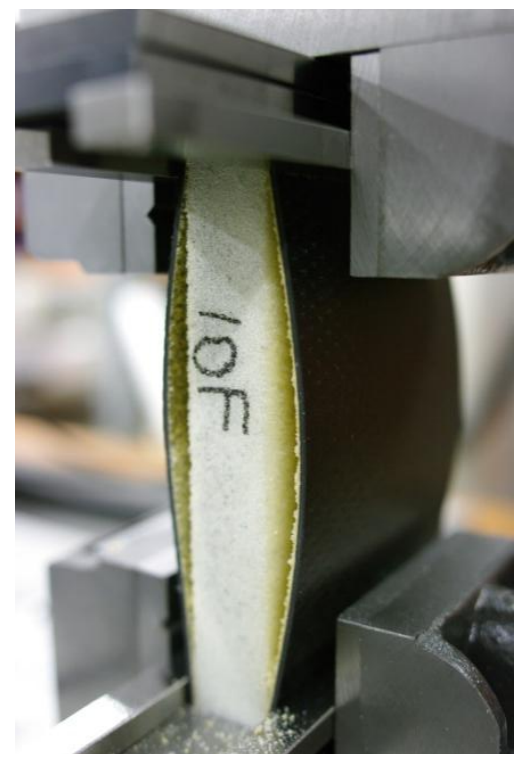

Figure 7. Observed failure mode for EWC samples with oxidation.

\section{Experimental and Analytical Investigation of 3DFRFC}

The remainder of this paper describes current efforts that are focused on expanding the methodology developed for the Rohacell foam to the fabrication, NDE, and testing of a 3DFRFC sandwich composite with and without defects to obtain, experimentally, the effects of defects on the failure of a 3DFRFC sandwich composite. 3DFRFC's are next generation materials consisting of foam reinforced with rigid composite rods, Figure 8. The goal is to increase the performance of sandwich structures in extreme temperature ranges. 3DFRFC materials have garnered significant attention from the aerospace industry, and they are already being used in other industries. When primary structural components of launch vehicles are manufactured using this new technology it is expected that defects will occur inadvertently during manufacturing and handling. 


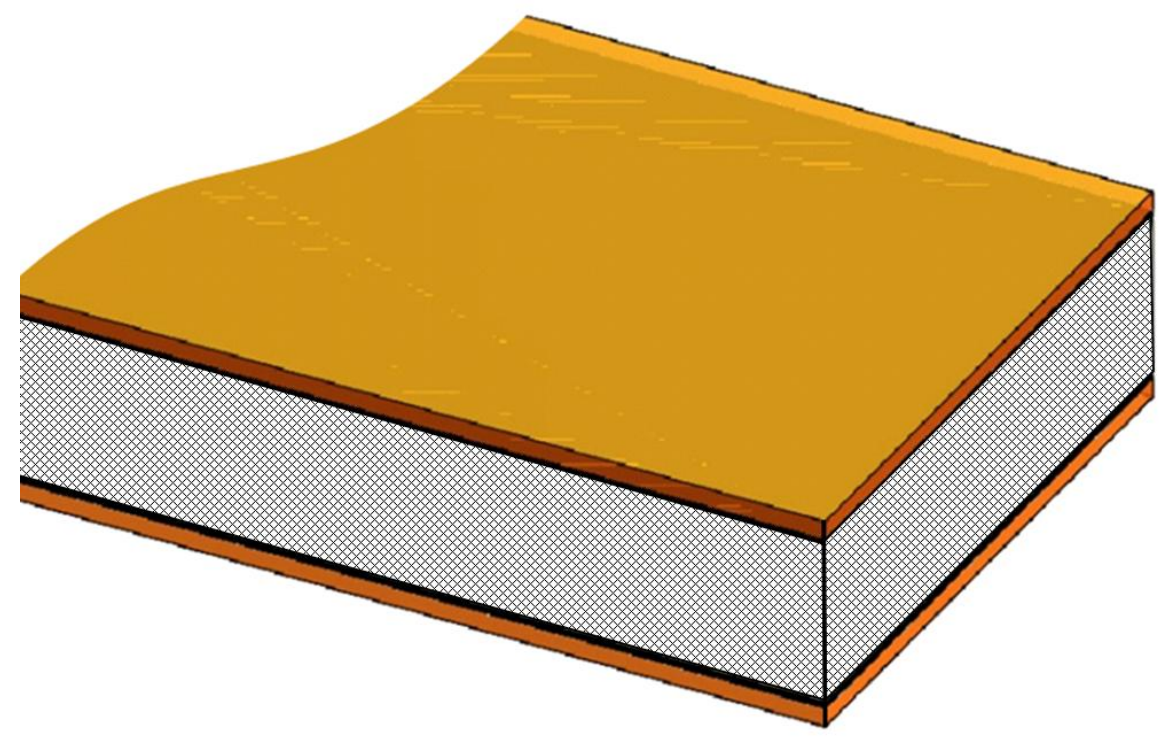

Figure 8. Illustration of composite sandwich structure made with 3DFRFC.

The material systems chosen for this investigation include the same AF191 film adhesive and IM7/8552 carbon epoxy for the facesheets that were used in the foam core study, but replaces the monolithic foam with a 0.5 inch thick $12 \mathrm{lbf} / \mathrm{ft}^{3}$ density 3DFRFC, the same density as the Rohacell 200WF. As with the monolithic foam core sandwich panels, the 3DFRFC sandwich panels were designed for testing in edgewise compression in accordance with ASTM C 364-99, but the specimen geometry deviated from the geometry used in the monolithic foam study due to dimensions of the available 3DFRFC panels and the difference in core thickness. The specimens were designed to contain circular debonds of 0.5 inch, 1.0 inch, 1.5 inch, or 2.0 inch diameter, Figure 9, which are representative of defect criteria used in the aerospace industry. The desired debonds were manufactured by removing a circular region of adhesive and replacing it with a PTFE insert, Figure 9. The panels were inspected via NDE to ensure panel quality and to verify debond placement in the cured sandwich panel prior to removing the desired samples from the fabricated panels, Figure 10. The 3DFRFC sandwich coupons were tested using the same procedures outlined for the monolithic foam core sandwich coupons.

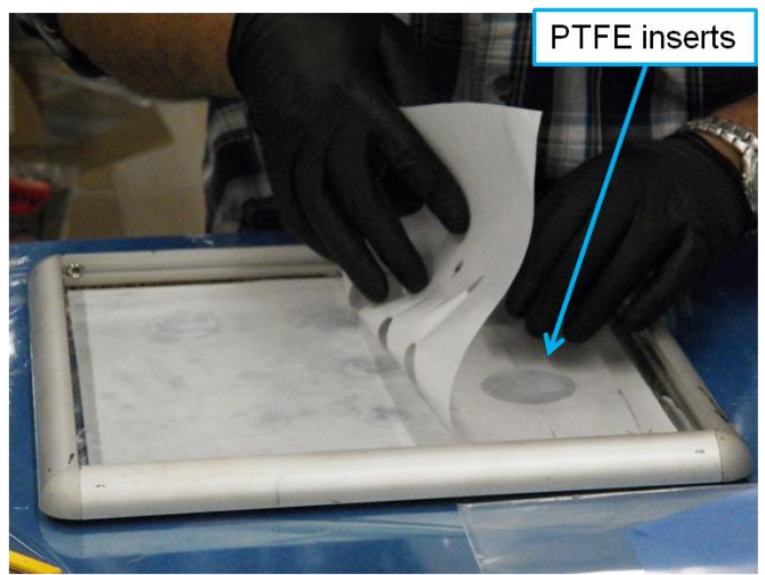

Figure 9. Film adhesive with various PTFE inserts. 


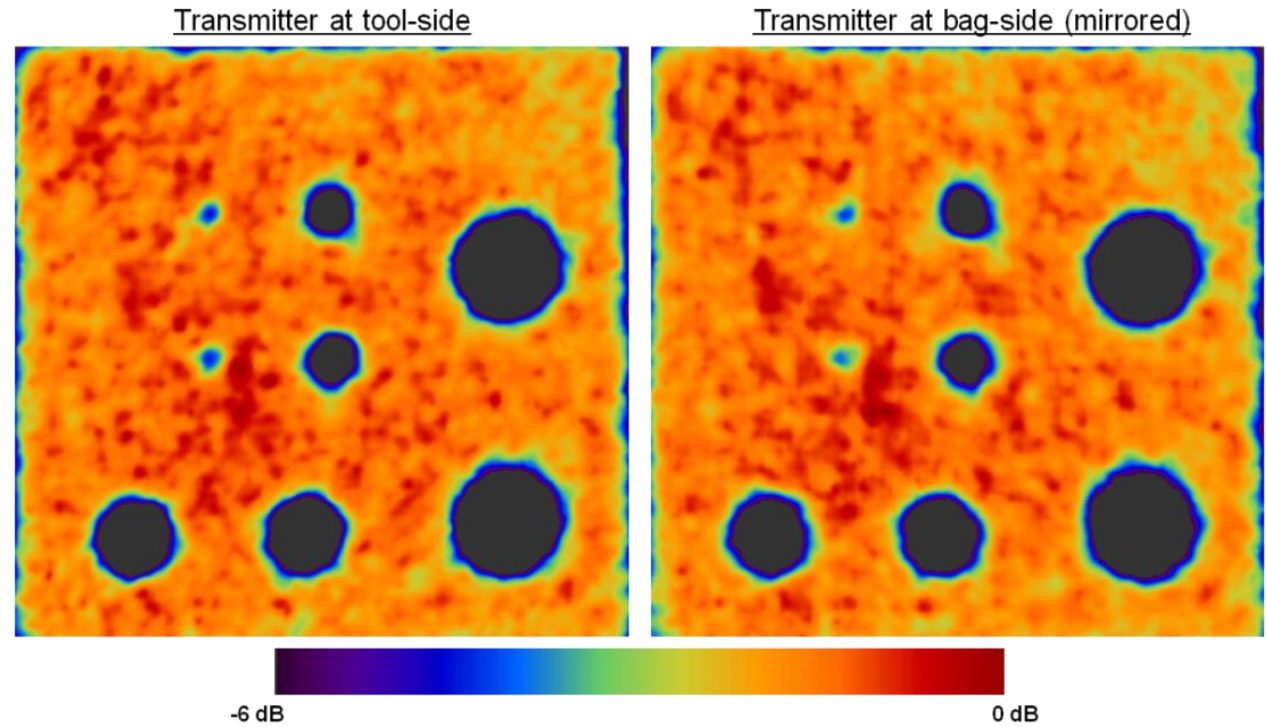

Figure 10. Ultrasonic through-transmission showing $0.5 \mathrm{inch}, 1.0 \mathrm{inch}, 1.5 \mathrm{inch}$, and 2.0 inch debonds.

Similar to the monolithic foam sandwich panels, the analytical evaluation was performed using finite element analysis. A quarter symmetry finite element model of the experimental setup was developed, and solved using the finite element software Abaqus to evaluate debonds between the facesheet and the core. As with the monolithic foam models, the facesheet plies were individually modeled with brick elements and the adhesive layer between the facesheet and the 3DFRFC was modeled with decohesion elements, Figure 11. No published experimental fracture properties exist for the 3DFRFC sandwich structures. As a result, the strength and fracture properties for the interface were modeled by using the previously measured values for the Rohacell $200 \mathrm{WF}$ foam as an approximate lower bound since it has the same bulk density. This modeling effort also takes advantage of recently developed micromechanics models ${ }^{7}$ for metallic and pin reinforced foams that models the reinforcements within the foam as beams on an elastic foundation to derive the effective orthotropic elastic properties for the 3DFRFC based on its specific microstructure and material composition.
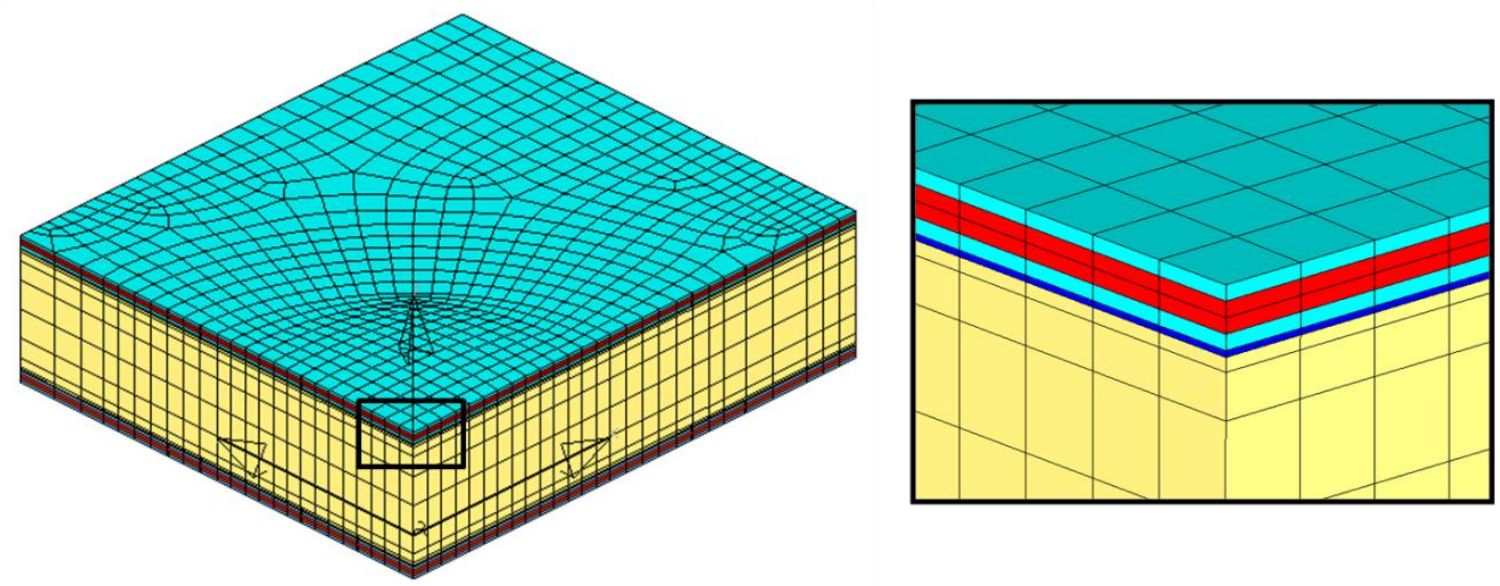

Figure 11. Abaqus quarter symmetry edgewise compression finite element model.

One challenge of applying the methods developed for foam core sandwich structures to the 3DFRFC is that decohesive zones yield accurate results when the crack plane is well defined ${ }^{8-10}$ but lose fidelity as the material becomes more discretized and the crack path can no longer be inferred a priori. The added paths for load transfer in the 3D fiber reinforced foam core act to impede crack propagation within the foam and the use of decohesive elements to model this material will likely become less accurate and unable to capture the highly discretized nature of the 3DFRFC, particularly in situations with more complex loading. In the current study, the discrete cohesive 
zone model (DCZM) pioneered by Xie and Waas ${ }^{10}$ is used for modeling delamination between the 3DFRFC and the facesheets due to its increased modeling efficiency.

The failure load was predicted using nonlinear analysis with a progressive failure methodology. Boundary conditions were simulated by specifying displacements, thus enabling the numerical simulation of failure progression. Progressive failure analysis (PFA) did not account for the simulation of matrix-cracking and fiber failure because it was observed in the experiment that the structural response was linear. Failure of the samples occurred suddenly in the form of catastrophic facesheet compression failure. There was no indication that matrixcracking preceded the ultimate failure, but most likely it occurred concurrently with the onset of fiber failure. While PFA was used to simulate delamination propagation, the facesheet compression failure was predicted by identifying the load at which the facesheet stress exceeded its compression strength.

Linear buckling analysis was performed for each defect size to determine critical buckling modes. The linear buckling analysis showed that the primary critical buckling mode is a localized buckle of the facesheet adjoining the unbonded region. Subsequently, the modes from the linear buckling analysis were scaled to introduce a geometric imperfection into the nonlinear model. The magnitude of the imperfection introduced was varied from $0.01 \%-1.0 \%$ of the facesheet thickness. The analysis showed that the 1.5 inch debond model was highly sensitive to imperfections, resulting in a transition from pure facesheet compression failure to a buckling driven compression failure. For the $1.5 \mathrm{inch}$ and 2.0 inch debonds, the failure involves facesheet buckling induced compression failure that occurs concurrently with delamination initiation, Figure 12. Additionally, the values for the Mode II and mode III critical strain energy release rates were varied from 1 to 10 times the Mode I value and the predicted failure was found to be insensitive to the changes. This finding may not be applicable for a different composite system.
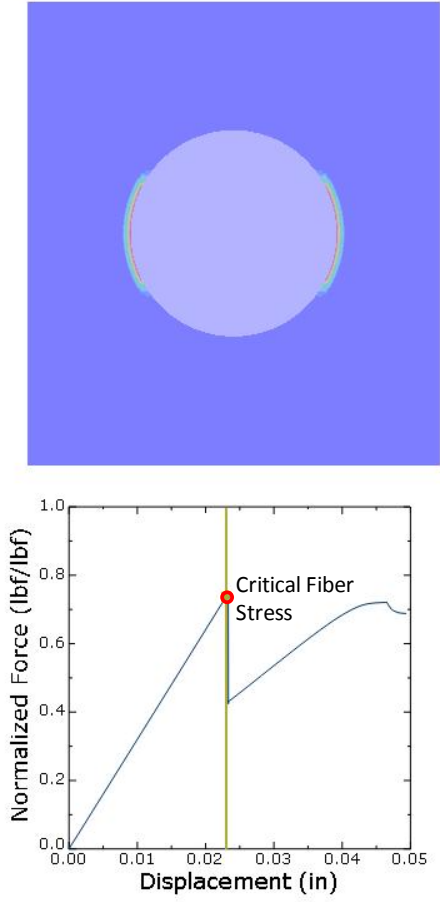

Figure 12. Failure analysis for 2.0 inch debond in 3DFRFC.

The results of the edgewise compression testing indicated that the strength was unaffected by the presence of 0.5 inch debonds. In these samples the fiber failure occurs before the onset of buckling. The strength for the remaining samples with debonds was reduced when compared to the sample without defect, especially for the 2.0 inch sample where an 18 percent strength reduction was observed, Table III. Fiber failure initiated with the onset of buckling for samples with 2.0 inch debonds. The analytical model for the 3DFRFC sandwich with the 2.0 inch debond predicts a larger effect due to delamination growth. This is possibly attributed to the lower bound on the fracture energy determined for Rohacell 200WF. It is expected that the model will be capable of capturing this failure mode more accurately once test data for the fracture properties for 3DFRFC have been obtained during subsequent research efforts. Additionally, a small reduction in strength was observed for 1.0 inch debonds. It is suspected that a tertiary 
failure mode is being induced due to local stress concentrations at the pins and the disruption of the loading paths of the pins caused by the presence of the debond. Local compressive pin failures observed in 1.0 inch samples near the failure point support this hypothesis. Further research into the cause of the observed strength reduction is planned using full surface strain capture mapping using digital image correlation.

Table III. Observed and predicted strength reduction with facesheet-core debonds.

\begin{tabular}{|c|c|c|c|c|}
\hline $\begin{array}{c}\text { Debond Diameter } \\
\text { (inch) }\end{array}$ & $\begin{array}{c}\text { Number of } \\
\text { Samples Tested }\end{array}$ & $\begin{array}{c}\text { Test Standard } \\
\text { Deviation (\%) }\end{array}$ & $\begin{array}{c}\text { Strength Reduction } \\
\text { Measured from Test (\%) }\end{array}$ & $\begin{array}{c}\text { Predicted Strength } \\
\text { Reduction }(\%)\end{array}$ \\
\hline- & 3 & 3.2 & $--*$ & $0.0^{*}$ \\
\hline 0.5 & 1 & NA & $0.0^{*}$ & $0.0^{*}$ \\
\hline 1.0 & 4 & 1.0 & $5.9^{*}$ & $0.0^{*}$ \\
\hline 1.5 & 3 & 5.4 & $6.5^{*}$ & $0.0^{*}-9.5^{* *}$ \\
\hline 2.0 & 4 & 1.3 & $19.2^{* *}$ & $22.6^{* *}-32.0^{* *}$ \\
\hline
\end{tabular}

Failure Mode: Facesheet Compression* Localized Buckling of Facesheet ${ }^{* * *}$

Additional insight into the material behavior and the interaction of the discrete constituents of the 3DFRFC sandwich will be gained through the use of digital image correlation. This capability will allow for the mapping of the two dimensional strain fields on the surface of the specimen throughout the test. Furthermore, multiple surfaces can be mapped simultaneously allowing for the full-field strains on the facesheet of a sample containing a defect to be captured simultaneously with the strain field of sample edge during an edgewise compression test. This capability will provide critical insight and allow for a deeper understanding of the microstructure interaction of the 3DFRFC and aid in the development of models capable of capturing this interaction.

\section{Conclusion}

A manufacturing, test, analysis, and NDE program was developed to address a common manufacturing discontinuity that has occurred in composite sandwich structures, facesheet-to-core debonds. The preliminary results of the experimental investigation of the failure of 3DFRFC sandwich composites, their sensitivity to debonds, and the ability of standard modeling practices to predict their failure was presented. While the monolithic foam sandwich structure study was used to develop the methodology used for the 3DFRFC sandwich structure and these preliminary results suggest the 3DFRFC demonstrates greater strength reduction with the presence of larger debonds under ambient conditions, direct comparative studies will be needed to validate any trends due to the differences in core thickness, sample size, and cure schedules between the two materials presented; however, comparative studies are planned. The outcomes of this continued research will provide the critical understanding and engineering tools required to fully exploit the benefits of advanced three-dimensionally reinforced sandwich structures in current and future spacecraft and launch vehicles and have transformative impacts to the ability to utilize advanced materials in commercial aerospace and non-aerospace applications.

\section{Acknowledgments}

The authors gratefully acknowledge the technical support on this research from James Tuck-Lee, Thomas Albright, James Nokes, and Neil Ives from The Aerospace Corporation and Wooseok Ji and Paul Davidson from the University of Michigan. This work was supported by a NASA Office of the Chief Technologist's Space Technology Research Fellowship.

\section{References}

${ }^{1}$ NidaFusion SXO/SXF, http://www.nida-core.com/spanish/fusiprod_sxosxf.htm, 2006-2008, Nida-Core Corporation.

${ }^{2}$ TYCORE® Products, http://www.webcoreonline.com/tycor\%C2\%AE-products, 2011 WebCore Technologies.

${ }^{3}$ ASTM Standard C364, 1999, "Standard Test Method for Edgewise Compressive Strength of Sandwich Constructions," ASTM International, West Conshohocken, PA, 1999, 10.1520/C0364_C0364M-99, www.astm.org.

${ }^{4}$ Zenkert, D., Shipsha, A., and Burman, M., "Fatigue of Closed Cell Foams," Journal of Sandwich Structures and Materials, 8, 2006, pp. 517-538.

${ }^{5}$ Goyal, V., J. Rome, P. Schubel, J. Tuck-Lee, G. Steckel, D. Patel. 2010. "Strength Evaluation of Foam Core Sandwich Structures with Butt Joints," $25^{\text {th }}$ Annual Technical Conference of the American Society for Composites. September 20-23, 2010. Dayton, $\mathrm{OH}$.

63M Scotch-Weld Structural Adhesive Film AF 191. March 2009. Technical Datasheet, St. Paul, MN. 
${ }^{7}$ Liu, T., Deng, Z., and Lu,T., “Analytical Modeling and Finite Element Simulation of the Plastic Collapse of Sandwich Beams with Pin-reinforced Foam Cores", International Journal of Solids and Structures, 45, 2008, pp. 5127-5151.

${ }^{8}$ Alfano, G. and Crisfield, M., "Finite Interface Elements for the Delamination Analysis of Laminated Composite Structures: Mechanical and Computational Issues," Journal for Numerical Methods in Engineering, 50, 2001, pp. 1701-1736.

${ }^{9}$ Goyal, V., Johnson, E., and Dávila, C., "Irreversible Constitutive Law for Modeling the Delamination Process Using Interfacial Surface Discontinuities," Composite Structures, 65, 2004, pp. 289-305.

${ }^{10}$ Xie, D., Waas, Anthony M., "Discrete cohesive zone model for mixed-mode fracture using finite element analysis," Engineering Fracture Mechanics, 73, 2006, pp. 1783-1796. 